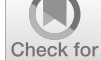

Check for

updates

Cite as

Nano-Micro Lett.

(2021) 13:42

Received: 28 September 2020

Accepted: 19 November 2020

Published online: 4 January 2021

(C) The Author(s) 2021

\section{Armoring Black Phosphorus Anode with Stable Metal-Organic-Framework Layer for Hybrid K-Ion Capacitors}

\author{
Mengzhu Xu ${ }^{1,2}$, Yutong Feng ${ }^{1,2}$, Bingjie Chen ${ }^{2}$, Ruijin Meng ${ }^{2}$, Mengting $\mathrm{Xia}^{2}$, Feng Gu ${ }^{3}$, \\ Donglei Yang ${ }^{4}$, Chi Zhang ${ }^{1,2}$, Jinhu Yang ${ }^{1,2} \bowtie$
}

\title{
HIGHLIGHTS
}

- The ultrathin metal-organic-framework (MOF) interphase layer with high mechanical/chemical stability was in situ grown on black phosphorus nanosheets (BPNSs).

- MOF interphase layers as an ordered porous and robust protective layer can facilitate $\mathrm{K}$ ion diffusion and accommodate the volume change of the electrode.

- Benefiting from the improved reaction kinetics and enhanced electrode stability, the BPNS@MOF anode for potassium-ion capacitors exhibits outstanding cycle performance.

\begin{abstract}
Potassium-ion capacitors (KICs) are promising for sustainable and eco-friendly energy storage technologies, yet their slow reaction kinetics and poor cyclability induced by large $K$-ion size are a major obstacle toward practical applications. Herein, by employing black phosphorus nanosheets (BPNSs) as a typical high-capacity anode material, we report that BPNS anodes armored with an ultrathin oriented-grown metal-organic-framework (MOF) interphase layer (BPNS@ MOF) exhibit regulated potassium storage behavior for highperformance KICs. The MOF interphase layers as protective layer with ordered pores and high chemical/mechanical stabil-
\end{abstract}

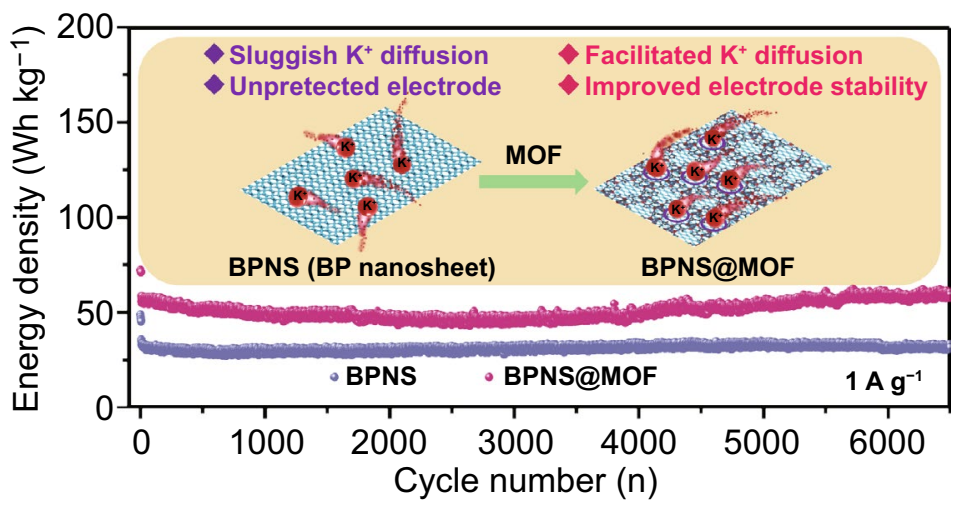
ity facilitate $K$ ion diffusion and accommodate the volume change of electrode, beneficial for improved reaction kinetics and enhanced cyclability, as evidenced by substantial characterizations, kinetics analysis and DFT calculations. Consequently, the BPNS@MOF electrode as KIC anodes exhibits outstanding cycle performance outperforming most of the reported state-of-art KICs so far.

KEYWORDS Black phosphorus; MOF; Interphase engineering; Potassium-ion capacitor; Cycling stability

Mengzhu $\mathrm{Xu}$ and Yutong Feng contributed equally to this work

$\triangle$ Chi Zhang, chizhang@ $@$ tongji.edu.cn; Jinhu Yang, yangjinhu@tongji.edu.cn

1 Research Center for Translational Medicine \& Key Laboratory of Arrhythmias of the Ministry of Education of China, East Hospital, Tongji University School of Medicine, Shanghai 200120, People's Republic of China

2 School of Chemical Science and Engineering, Tongji University, Shanghai 200092, People's Republic of China

3 Institute for Process Modelling and Optimization, Jiangsu Industrial Technology Research Institute, SIP, 388 Ruoshui Road, Suzhou, Jiangsu, People's Republic of China

4 Institute of Molecular Medicine School of Medicine, Shanghai Jiao Tong University, Shanghai 200127, People's Republic of China 


\section{Introduction}

Recently, potassium-based electrochemical energy storage devices have attracted ever-increasing attention due to the natural abundance of potassium element and the low redox potential of $\mathrm{K} / \mathrm{K}^{+}\left(-2.92 \mathrm{~V}\right.$ vs SHE) close to that of $\mathrm{Li} / \mathrm{Li}^{+}$ $(-3.04 \mathrm{~V}$ vs SHE) [1-3]. So far, potassium $(K)$-ion batteries (KIBs) are the most attracting potassium-based electrochemical energy storage device and extensive research efforts have been carried out [4, 5]. However, similar to other alkali metal ion batteries, KIBs suffer from inferior cycling lifespan and insufficient power density [6]. On the other hand, hybrid potassium $(K)$-ion capacitors (KICs) have gained particular interest and are expected as the replacement for KIBs due to their unique characteristic that couples a capacitor-type cathode with a battery-type anode, which are expected to deliver outstanding energy at high power [7-15]. Thereinto, battery-type anodes associating with complicated potassiation/depotassiation behaviors are considered as the key component affecting the performance of KICs. To date, various candidate materials, such as carbon-based materials [7, 8], $\mathrm{V}_{2} \mathrm{C}$ MXene [9], $\mathrm{Ca}_{0.5} \mathrm{Ti}_{2}\left(\mathrm{PO}_{4}\right)_{3} @ \mathrm{C}[10], \mathrm{K}_{2} \mathrm{Ti}_{6} \mathrm{O}_{13}$ [11], organic $\mathrm{K}_{2} \mathrm{TP}$ [12], $\mathrm{Co}_{2} \mathrm{P}$ [13], N-MoSe 2 /G [14] and $\mathrm{MoS}_{2-\mathrm{x}} \mathrm{Se}_{\mathrm{x}} / \mathrm{C}$-HNTs [15], have been investigated as anodes for KICs. Despite much progress, the comprehensive performance of KICs based on these anode materials is still far from satisfying the requirements. Therefore, the exploration of new anode materials with regulated potassium storage behaviors is highly desired for the development of highperformance KICs.

Black phosphorus (BP) has been recognized as a superior electrode material for electrochemical energy storage systems, including organic and aqueous alkaline metal ion batteries [16, 17], Li-S batteries [18] and hybrid ion capacitors [19], due to its unique properties such as high carrier mobility and high energy storage capacity [20, 21]. As an alloy-based anode material, BP offers superior specific capacity (1154 $\mathrm{mAh} \mathrm{g}^{-1}$ for $\mathrm{K}_{4} \mathrm{P}_{3}$ ) [22, 23] compared to the traditional K-ion intercalation materials [24-26]. However, BP-based materials have not yet been explored in KICs. The most serious challenge is the dramatic volume expansion causing electrode pulverization induced by the large ion size of $\mathrm{K}^{+}\left(1.38 \AA\right.$ vs. $0.76 \AA$ of $\left.\mathrm{Li}^{+}\right)$[27]. In most cases, carbon-based materials were employed as the protective layers or matrix for anode materials to accommodate the volume expansion and keep the integrity of the electrodes. However, this strategy cannot be applicable to BP which possesses a low sublimation temperature $\left(530{ }^{\circ} \mathrm{C}\right)$ and is sensitive to water and oxygen, because the introduction of carbon materials was usually realized through hightemperature carbonization or hydrothermal procedures.

Zeolitic imidazolate frameworks (ZIFs) are a subclass of metal-organic frameworks (MOFs) and possess similar structures to zeolites. Among which, ZIF-8 is constructed from tetrahedrally coordinated zinc ions linked by organic dimethyl imidazole (Mim) units [28]. ZIF-8 has attracted significant interest for its easy preparation, ordered nanoporous structure, exceptional mechanical stability and ultrahigh chemical stability resulting from the metal-nitrogen bonds [29]. Notably, it was reported that ZIF-8 could withstand metal ion intercalation/deintercalation [30-32], preserving its structure/chemical composition intact during cycles. For example, Fan et al. introduced a stable artificial solid electrolyte interphase (SEI) film prepared by polyvinyl alcohol (PVA) cementing a metal-organic framework (Zn-MOF), which is beneficial for inhibiting dendrite growth and easing the volume change [30]. Li et al. reported the commercial $\mathrm{LiNi}_{1 / 3} \mathrm{Co}_{1 / 3} \mathrm{Mn}_{1 / 3} \mathrm{O}_{2}\left(\mathrm{NCM}_{333}\right)$ cathode modified by synthesizing ZIF-8 in situ on the surface of $\mathrm{NCM}_{333}$, in which ZIF-8 can not only act as a shield against the electrolyte corrosion, but also enable faster lithium-ion transfer at the interfacial regions [31]. Liu et al. proposed an in situ grown porous ZIF-8 as an ideal $\mathrm{Zn}^{2+}$ modulation layer to effectively regulate the aqueous $\mathrm{Zn}$ deposition behavior [32]. The unique properties make ZIF-8 a promising protective layer for BP.

In this work, ultrathin MOF interphase (MI) layer made of ZIF-8 has been constructed on the surface of black phosphorus nanosheets (BPNSs) by a in situ oriented-grown strategy and the fabricated composite material (denoted as BPNS@ MOF) is employed as representative anodes for KICs. The as-prepared BPNS@MOF exhibits a set of merits as anodes for KICs: (i) ultrathin MI layers as protective layer with ordered nanopores are beneficial to efficient $K$ ion transport; (ii) the high chemical and structural stability of ZIF-8 plus the robust interface can effectively accommodate the volume change of BP to prevent electrode pulverization; (iii) the thin thickness of the BPNSs can both shorten the diffusion length of $K$ ions and effectively reduce the large volume change of the electrode during charge/discharge processes. Accordingly, the hybrid KIC constructed by coupling the BPNS@ 
MOF anode and activated carbon (AC) cathode can deliver a significantly improved energy density compared with the BPNS-based KIC and, particularly, outstanding cycle performance outperforming most of the reported state-of-art KICs so far.

\section{Experimental Section}

\subsection{Synthesis of the BPNSs}

Prior to the synthesis, bulk BP crystals were firstly prepared according to the previously reported method [33] by using red phosphorus, tin and iodine as source materials sealed in a vacuumed ampule in a muffle furnace. The black phosphorus nanosheets were prepared by electrochemical cationic intercalation [34] and liquid exfoliation of the prepared bulk BP crystals. In a typical experiment, BP crystals $(8 \mathrm{~mm}$ in length, $5 \mathrm{~mm}$ in width) were used as the cathode and a Pt foil (10 $\mathrm{mm}$ in length, $10 \mathrm{~mm}$ in width) was employed as the counter electrode. The distance between the counter electrode and the working electrode was kept at $\approx 1.5 \mathrm{~cm}$. Static potentials of $-5 \mathrm{~V}$ were applied to the bulk BP electrodes using an electrochemical workstation (CHI 660e). The electrolyte was prepared by dissolving TBAB (16.1 g) in DMF (100 mL), and the exfoliation was lasted for $1 \mathrm{~h}$. Then, the materials were sonicated in an ice bath for $2 \mathrm{~h}$ at a power of $400 \mathrm{~W}$. Subsequently, the dispersions were separately washed with DMF and methyl 10 times to remove TBAB and DMF in the solution. Next, the materials were dispersed in methyl and the resultant brown suspension was centrifuged at $1000 \mathrm{rpm}$ for $10 \mathrm{~min}$ to remove the residual unexfoliated bulk BP. Afterward, the resultant BPNS dispersions were centrifuged for $10 \mathrm{~min}$ at $10,000 \mathrm{rpm}$ to collect the products, followed by drying in a vacuum oven at $60{ }^{\circ} \mathrm{C}$ for $12 \mathrm{~h}$.

\subsection{Synthesis of the BPNS@MOF}

In a typical procedure, $50 \mathrm{mg}$ of as-prepared few-layer BPNSs were dispersed into $150 \mathrm{~mL}$ of methanol containing $1.65 \mathrm{~g}$ of PVP (K29-32). After ultrasonic dispersion, $0.0745 \mathrm{~g}$ of $\mathrm{Zn}\left(\mathrm{NO}_{3}\right)_{2} \cdot 6 \mathrm{H}_{2} \mathrm{O}(0.25 \mathrm{mmol})$ was subsequently dissolved into the mixed solution and stirred for $2 \mathrm{~h}$ at room temperature. Then, $0.137 \mathrm{~g}(1.67 \mathrm{mmol})$ of 2-methylimidazole was added into the above solution (the molar ratio of
$\mathrm{Zn}\left(\mathrm{NO}_{3}\right)_{2} \cdot 6 \mathrm{H}_{2} \mathrm{O}: 2-\mathrm{Mim}$ was $\left.1: 6.68\right)$, followed by vigorous stirring for $4 \mathrm{~h}$ at room temperature. Finally, the product was collected by centrifugation and washed with methanol for three times, followed by drying in a vacuum oven at $60{ }^{\circ} \mathrm{C}$ overnight and then stored in a glove box filled with argon before use. In the typical synthesis, the product is denoted as BPNS@MOF. For comparison, BPNS@MOF2, BPNS@MOF-3 were also prepared through the same approach adding of 0.00298 and $0.149 \mathrm{~g} \mathrm{Zn}\left(\mathrm{NO}_{3}\right)_{2} \cdot 6 \mathrm{H}_{2} \mathrm{O}$, respectively, while the ratio of $\mathrm{Zn}\left(\mathrm{NO}_{3}\right)_{2} \cdot 6 \mathrm{H}_{2} \mathrm{O} / 2-\mathrm{Mim}$ remains unchanged.

\subsection{Material Characterization}

The morphologies of the as-prepared samples were observed using a scanning electron microscopy (SEM, Hitachi S-4800, $3 \mathrm{kV}$ ) and transmission electron microscope (TEM, Talos F200X G2) together with associated energy-dispersive X-ray spectroscopy (EDS). Atomic force microscopy (Dimension ICON with NanoScope V controller, Bruker, USA) was used to characterize the samples in tapping mode in air. Samples were dropped onto a thermally oxidized $\mathrm{Si}(300 \mathrm{~nm} \mathrm{SiO}$ ) substrate for AFM measurement. X-ray diffraction patterns (XRD) were recorded on a D8 advance X-ray diffractometer with a $\mathrm{Cu} \mathrm{K} \alpha$ radiation source $(\lambda=0.15418 \mathrm{~nm})$ in a step of $0.02^{\circ}$ over a $2 \theta$ range of $5-70^{\circ}$. TriStarII 3020 system is used to characterize nitrogen adsorption-desorption isotherms, the sample was degassed in vacuum at $200{ }^{\circ} \mathrm{C}$ for at least $4 \mathrm{~h}$. The Brunauer-Emmett-Teller (BET) method was utilized to calculate the surface areas. The pore size distributions were retrieved by using the Barrett-Joyner-Halanda (BJH) method from the adsorption branch of the isotherms. $\mathrm{X}$-ray photoelectron spectroscopy (XPS) investigation was conducted on PHI-5000C ESCA system (Perkin Elmer) with $\mathrm{Mg} \mathrm{K} \alpha$ radiation, and the binding energies (BEs) were referred of the $\mathrm{C} 1 \mathrm{~s}$ peak at $284.80 \mathrm{eV}$. Raman spectra were measured by using a spectrophotometer (Invia, Renishaw, Germany) with a $514 \mathrm{~nm}$ laser. Fourier transform infrared spectra were recorded on Nicolet 6700 FT-IR spectrometric analyzer.

\subsection{Electrochemical Measurements}

For fabrication of the anode electrodes, the active material (BPNSs or BPNS@MOF), carbon black additive and 
poly(acrylic acid) (PAA) binder were prepared into a slurry in N-methyl-2-pyrrolidone (NMP) with a weight ratio of 7:2:1, followed by pasting the slurry onto a copper foil current collector. The areal mass loading of the anode is about $1.2-1.5 \mathrm{mg} \mathrm{cm}^{-2}$. For fabrication of the cathode electrodes, the active carbon (AC), polyvinylidene fluoride (PVDF) binder and carbon black additive were mixed with a weight ratio of $8: 1: 1$, followed by pasting the slurry onto an aluminum foil current collector. The electrodes were dried at $80{ }^{\circ} \mathrm{C}$ for $24 \mathrm{~h}$ under vacuum before the assemble of cells. Both hybrid KICs and half-cells of the KIBs were assembled inside an Ar filled glovebox where the oxygen and water concentrations are kept below $0.01 \mathrm{ppm}$. Hybrid KICs were assembled with CR2032 coin cells, employing a glass fiber filter (Whatman GF/D) as the separator, $1.0 \mathrm{~mol} \mathrm{~L}^{-1} \mathrm{KPF}_{6}$ in ethylene carbonate:diethyl carbonate (1:1, volume ratio) as the electrolyte. Before assembling the devices, the BPNS@ MOF (or BPNS) anode electrodes were preactivated in halfcells by charging/discharging at $50 \mathrm{~mA} \mathrm{~g}^{-1}$ for 5 cycles. Then, the cells were disassembled inside the glove box and a KIC was assembled employing the preactivated anode and activated carbon cathode. The anode to cathode mass ratio was 4:1. For comparison, KICs with different anode to cathode mass ratio of 3:1, 5:1 were also prepared. Half-cells of the KIBs were assembled with CR2016 coin cells, employing a glass fiber filter (Whatman $\mathrm{GF} / \mathrm{F}$ ) as the separator, $1.0 \mathrm{~mol} \mathrm{~L}^{-1} \mathrm{KFSI}$ in ethylene carbonate:diethyl carbonate $(1: 1$, volume ratio) as the electrolyte, $K$ metal as the counter electrode. All the electrochemical tests were carried out at room temperature. Galvanostatic discharge-charge cycling was performed using a Land-2001A (Wuhan, China) automatic battery tester. Cyclic voltammetry was performed on an electrochemical workstation (CHI 660e) at a scan rate of $0.01 \mathrm{mV} \mathrm{s}^{-1}$, and the electrochemical impedance spectra were recorded using the same electrochemical workstation by applying an AC voltage of $5 \mathrm{mV}$ amplitude over the frequency range from $100 \mathrm{kHz}$ to $0.01 \mathrm{~Hz}$. The gravimetric energy and the power densities of the KIC device were calculated by numerically integrating the galvanostatic discharge profiles using the equations as follows:

$$
\begin{aligned}
& E_{\text {mass }}=\int_{t 2}^{t 1} I U / m d t \\
& P_{\text {mass }}=E_{\text {mass }} / t
\end{aligned}
$$

where $I$ is the constant current $(A), U$ is the working voltage $(V), m$ is the total mass of the electrode, $t_{1}$ and $t_{2}$ are the start/end-of-discharge time $(s)$ of the KIC device, respectively, and $t$ is the discharge time $(s)$.

\subsection{Theoretical Calculation}

DFT calculations were performed using the Vienna ab initio simulation package. The exchange-correlation energy was described by the generalized gradient approximation (GGA) proposed by Perdew, Burke and Ernzerhof (PBE) [35]. The electronic wave functions were expanded using a plane wave basis set with a kinetic energy cutoff of $400 \mathrm{eV}$ with a $2 \times 2 \times 1$ Monkhorst-Pack sampled k points. The model was built by using the (020) plane of black phosphorus, the (112) plane of ZIF-8 and the (002) plane of $\mathrm{K}_{2} \mathrm{SO}_{4}$. To find out the minimum energy path (MEP) and saddle points between the initial and final positions and calculate the migration energy barrier for $\mathrm{K}$ diffusion, the climbing image nudged elastic band (CI-NEB) method was employed [36].

\section{Results and Discussion}

\subsection{Synthesis and Characterizations of the BPNS@ MOF}

The overall synthesis process for the BPNS@MOF samples is presented in Fig. 1a. Briefly, the BPNSs were obtained through the exfoliation of bulk BP by electrochemical cationic intercalation [34] (Step 1). Subsequently, the asexfoliated BPNSs were coated with a thin layer of polyvinylpyrrolidone (PVP) and abundant $\mathrm{Zn}$ ions on the surface through the electrostatic interaction in the presence of PVP and $\mathrm{Zn}\left(\mathrm{NO}_{3}\right)_{2}$ in methanol solution, consecutively (Step 2). Then, in situ growth of ZIF-8 layer on surface of the BPNSs was triggered by the addition of the ligand of 2-methylimidazolate [37], forming a crystalline MI layer with ordered pores on the BPNSs as well as the final product of the BPNS@MOF (Step 3).

Figure 1b, c shows TEM images of the as-exfoliated BPNSs and the typical BPNS@MOF, respectively. The BPNSs display a typical nanosheet-like shape and smooth surface, with the diameter up to several micrometer (Figs. 1b and S1a, b). A high-resolution TEM (HRTEM) image (inset in Fig. 1b) displays clear lattice fringes with d-spacings of 

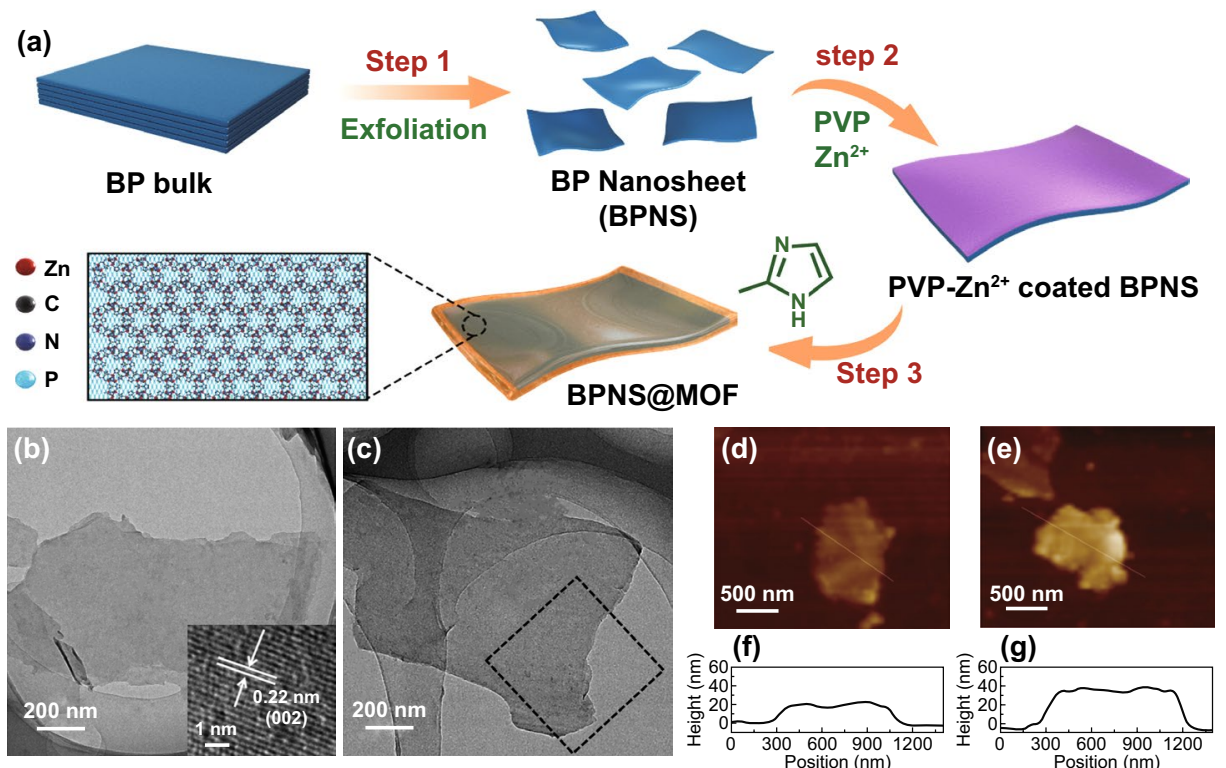

(h)
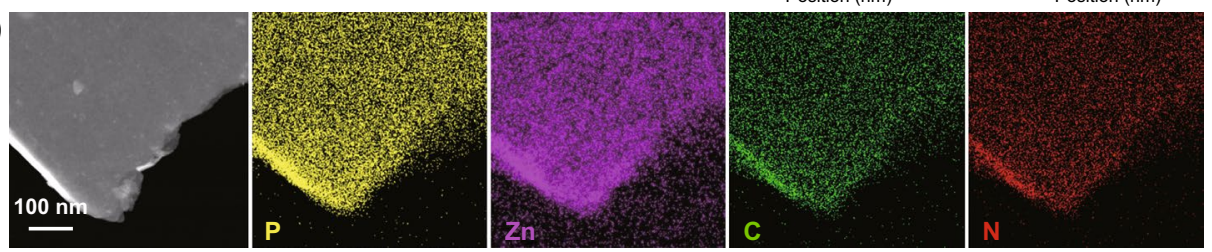

Fig. 1 a Schematic illustration of the formation process of the BPNS@MOF. b, c TEM images of the pristine BPNSs (b) and BPNS@MOF (c). The inset in (b) is a HRTEM image showing crystal lattices for the BPNSs. The black dotted box in (c) corresponds to the area of element mappings in (h). d, e AFM images of BPNSs (d) and BPNS@MOF (e). f, g Height profiles along the white lines in (d) and (e). h Scanning TEM image and the corresponding element mappings of P, Zn, C and N elements of the BPNS@MOF

$\approx 0.22 \mathrm{~nm}$, corresponding to the (002) plane of BP (JCPDS No. 76-1957) [38], which also confirms the top face of the BPNSs is bounded by the $\{020\}$ planes. The BPNS@MOF holds a similar nanosheet-like morphology to the pristine BPNSs (Figs. 1c and S1c, d) and a much thicker thickness $(\sim 34 \mathrm{~nm})$ relative to the BPNSs $(\sim 20 \mathrm{~nm})$ revealed by the corresponding atomic force microscopy (AFM) line scan image (Fig. 1d-g), indicating the thickness of the MI layer in the BPNS@MOF is about $7 \mathrm{~nm}$. In addition, the corresponding element mappings in Fig. $2 \mathrm{~h}$ (the area enclosed by the dotted line in Fig. 2c) demonstrate that P, Zn, C and $\mathrm{N}$ elements are uniformly distributed throughout the whole nanosheet, indicating the successful growth of a MI layer on the BPNSs with high uniformity. Although the MOF layer definitely exists and uniformly distribute on BPNSs as evidenced by AFM and element mapping, it cannot be directly observed by TEM at a high magnification (Fig. S2) as the MOF is crystalline. The mass percentage of MOF and BP in the typical BPNS@MOF sample was measured to be
45.51 and $54.49 \%$, respectively, by inductive coupled plasma emission spectrometer (ICP-MS). For comparison, BPNS@ MOF samples with different MOF contents, i.e., a lower content of $25.05 \%$ and a higher content of $56.11 \%$ were also prepared and denoted as BPNS@MOF-2 and BPNS@MOF3, respectively (Figs. S3a-d and S4). The BPNS@MOF-2 and BPNS@MOF-3 also show nanosheet-like morphology similarly to the BPNSs and BPNS@MOF, yet distinct from ZIF-8 polyhedral nanocrystals synthesized in the absence of the BPNSs (Fig. S3e, f). It is found that for the BPNS@ MOF-3, obvious ZIF-8 nanoparticles with sizes of 20-50 nm appear on BPNSs, implying the self-nucleation of ZIF-8 was occurred due to the high MOF content.

Fourier transform infrared spectrometer (FTIR) spectra in Fig. 2a prove the successful preparation of the BPNS@MOF. For the BPNSs, no apparent peak is observed in the FTIR spectrum, indicating that the BP is infrared inactive. For the BPNS@MOF, all peaks match well with ZIF-8 derived from various vibration modes [39]. Specifically, the intense 

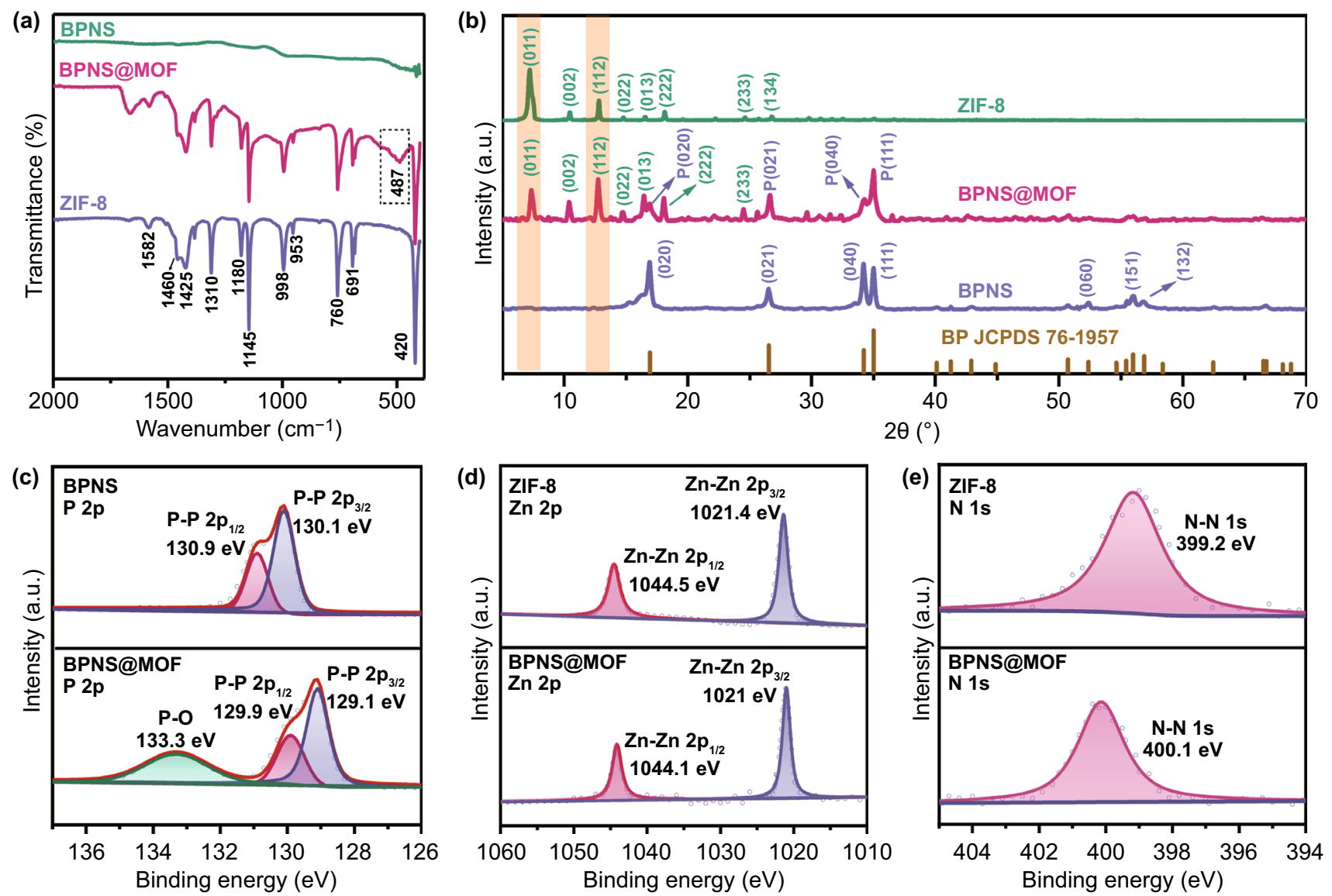

Fig. 2 a FTIR spectra of the BPNSs, BPNS@MOF and ZIF-8. b XRD patterns of the BPNSs, BPNS@MOF and ZIF-8. c High-resolution P 2p XPS spectra of the BPNSs and BPNS@MOF. d Zn 2p and e N 1 s XPS spectra of ZIF-8 and BPNS@MOF

vibrational bands at $1350-1500 \mathrm{~cm}^{-1}$ are associated with the entire ring stretching vibrations of the imidazole moiety and the bands in the region of $900-1350 \mathrm{~cm}^{-1}$ are ascribed to the in-plane bending of the imidazole ring. The peak at $420 \mathrm{~cm}^{-1}$ can be assigned as the $\mathrm{Zn}-\mathrm{N}$ stretch mode [39], which confirms the coordination between metal clusters and organic linkers in the framework. The peak at $487 \mathrm{~cm}^{-1} \mathrm{can}$ be ascribed to the $\mathrm{Zn}-\mathrm{P}$ bond vibrations [40].

It should be noted that no peak corresponds to PVP in the FTIR spectrum of the BPNS@MOF, proving that PVP was completely removed after washing process. The $\mathrm{N}_{2}$ adsorption-desorption isotherms in Fig. S5a show the specific surface area $\left(\mathrm{S}_{\mathrm{BET}}\right)$ of the BPNS@MOF is $\sim 278.8 \mathrm{~m}^{2} \mathrm{~g}^{-1}$ with pore sizes in the range of $1-3 \mathrm{~nm}$. Obviously, the $S_{\text {BET }}$ value was significantly increased after coating MI layer on the BPNSs $\left(\mathrm{S}_{\mathrm{BET}}=6.1 \mathrm{~m}^{2} \mathrm{~g}^{-1}\right)$.

Figure $2 \mathrm{~b}$ shows the XRD patterns of the BPNSs, BPNS@ MOF as well as ZIF-8 crystals. The XRD pattern of the
BPNS@MOF exhibits a set of diffraction peaks assigned to orthorhombic BP (JCPDS No. 76-1957) [38] and cubic ZIF-8 [41], respectively. The results indicate the MI layers are crystalline and also confirm the formation of the BPNS@ MOF. Interestingly, it is noted that the peak corresponding to the (011) plane is the strongest in pristine ZIF-8 crystal while the (112) peak become the strongest in the BPNS@ MOF, suggesting that the (112) plane becomes dominant in the MI layer and an oriented growth of (112) plane occurs during deposition of the MI layer. To probe the reason of the change of dominant plane, XPS examination was conducted for the BPNS@MOF, BPNSs and ZIF-8 (Figs. 2c-e and S5b-d). A survey XPS spectrum of the BPNS@MOF evidences the presence of $\mathrm{P}, \mathrm{Zn}, \mathrm{C}, \mathrm{N}$ and $\mathrm{O}$ in the material (Fig. S5b). The high-resolution $\mathrm{P} 2 \mathrm{p}$ spectrum of the BPNS@MOF displays two peaks at 129.1 and $129.9 \mathrm{eV}$ [42], showing a reduction of $1 \mathrm{eV}$ in binding energy compared with the BPNSs (Fig. 2c). In addition, the peaks for 
$\mathrm{Zn} 2 \mathrm{p}$ at 1021.0 and $1044.1 \mathrm{eV}$ also show a negative shift $(-0.4 \mathrm{eV})$ [43] compared with the ZIF-8 (Fig. 2d). While N $1 \mathrm{~s}$ of the BPNS@MOF exhibits a single peak at $400.1 \mathrm{eV}$ [44], which has a binding energy increase of $1 \mathrm{eV}$ relative to that of ZIF-8. Accordingly, the binding energy decrease of P 2p, Zn 2p and the increase of N 1 s for the BPNS@MOF is considered as a result of electron excursion from MOF to BPNS component via strong $\mathrm{Zn}-\mathrm{P}$ interfacial interaction. The strong $\mathrm{Zn}-\mathrm{P}$ interaction may influence the intrinsic growth habit of MOF, leading to the oriented growth of the (112) plane and the formation of the energy-stable interface of $\mathrm{BP}(020)-\mathrm{MOF}(112)$.

The priority of the MOF (112) plane orientation over the MOF (011) plane growing on the (020) plane of the BPNSs can be explained from two key points: zinc atom density and steric hindrance. Firstly, since the $\mathrm{Zn}-\mathrm{P}$ interaction is the direct force determining the orientation growth of MOF on the BPNSs, the plane holding a higher $\mathrm{Zn}$ atom density with a stronger Zn-P interaction will be preferentially formed at the interface between MOF and the BPNSs. Figure $3 a, b$ shows the top views of MOF (112) plane and MOF (011) plane cut out from single MOF unite cell. Calculations suggest that (112) plane has a higher Zn atom density than (011) plane (Table S1). We also analyze the $\mathrm{Zn}$ atom density of the (002), (013) and (222) planes which are most seen in the XRD pattern (Fig. S6). The result reveals that (112) plane has the highest $\mathrm{Zn}$ atom density among the above planes (Table S1). A higher $\mathrm{Zn}$ atom density with more $\mathrm{Zn}$ atoms in a given area of the MOF layer enables stronger $\mathrm{Zn}-\mathrm{P}$ interaction to form a stabler BP-MOF interface. Secondly, the (112) plane has no steric hindrance for $\mathrm{Zn}-\mathrm{P}$ interaction, yet the (011) plane suffers from a large steric hindrance. As shown in Fig. 3c, d, Zn atoms of the (112) plane are directly exposed and can approach $\mathrm{P}$ atoms to allow strong
$\mathrm{Zn}-\mathrm{P}$ interaction, while $\mathrm{Zn}$ atoms of the (011) plane are hindered by imidazole rings, which makes it difficult to directly interact with $\mathrm{P}$ atoms of the BPNSs. Take all into account, the MI layer with (112) plane dominant was induced by the strong $\mathrm{Zn}-\mathrm{P}$ interaction, which results in the stable interface of $\mathrm{BP}(020)-\mathrm{MOF}(112)$ that are crucial for high-performance electrochemical potassium storage.

\subsection{Electrochemical Measurements}

Electrochemical behaviors of the BPNS@MOF and BPNS as KIB anodes were investigated first. The cyclic voltammetry (CV) curves of the BPNS@MOF are shown in Fig. 4a. During the initial cathodic scan, a broad peak located at $0.4-0.75 \mathrm{~V}$ is ascribed to the decomposition of electrolyte and the formation of solid electrolyte interface (SEI) film. Two peaks located at 0.82 and $0.2 \mathrm{~V}$ correspond to the stepwise potassiation of $\mathrm{BP}$ to form the final $\mathrm{K}_{4} \mathrm{P}_{3}$ phase. In the anodic sweep, two peaks located at 0.78 and $1.24 \mathrm{~V}$ reveal the stepwise depotassiation process [22, 23]. Compared to the BPNS electrode (Fig. S7), CV curves of the BPNS@ MOF electrode are well overlapped in the subsequent cycles and show a higher reversibility. The HRTEM image (Fig. S8) coupling with cyclic voltammetry (CV) curves (Figs. 4a and S7) prove that both BPNS@MOF and BPNS anodes undergo the typical alloying reactions with $K$ ions and the final alloying product is $\mathrm{K}_{4} \mathrm{P}_{3}$. The charge-discharge curves of the BPNS@MOF electrode in Fig. 4b exhibits obvious plateaus and slope shape compared to that of the BPNS electrode (Fig. S9), indicating the sufficient depotassiation/ potassiation reaction of BP in the BPNS@MOF electrode. Specifically, a sloping region from 1.0-0.4 V followed by an inclined plateau from $0.4-0.1 \mathrm{~V}$ is observed in the discharge profile of the BPNS@MOF electrode, which corresponds
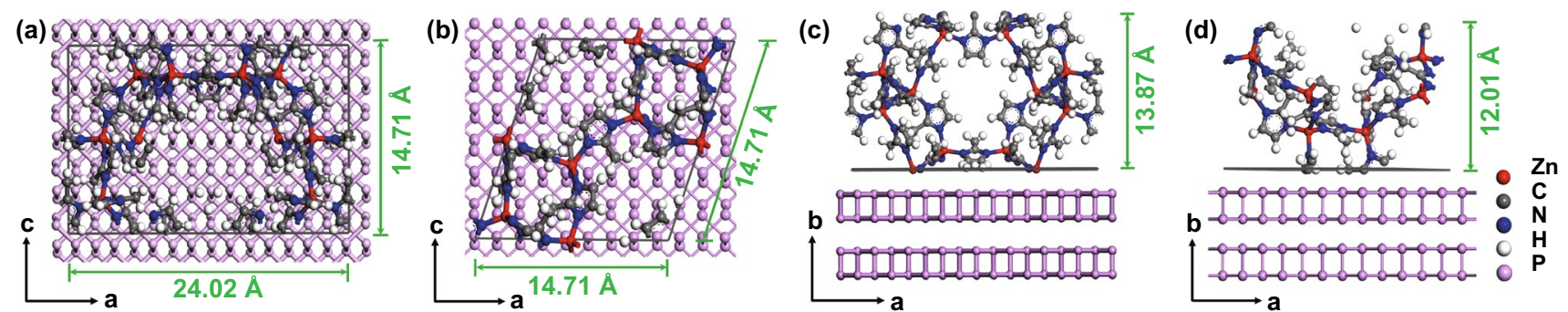

Fig. 3 Atomic arrangement of the (011) and (112) planes of a single MOF unit cell. a and b show top views of the (112) and (011) plane. c and d show side views of the (112) and (011) plane 

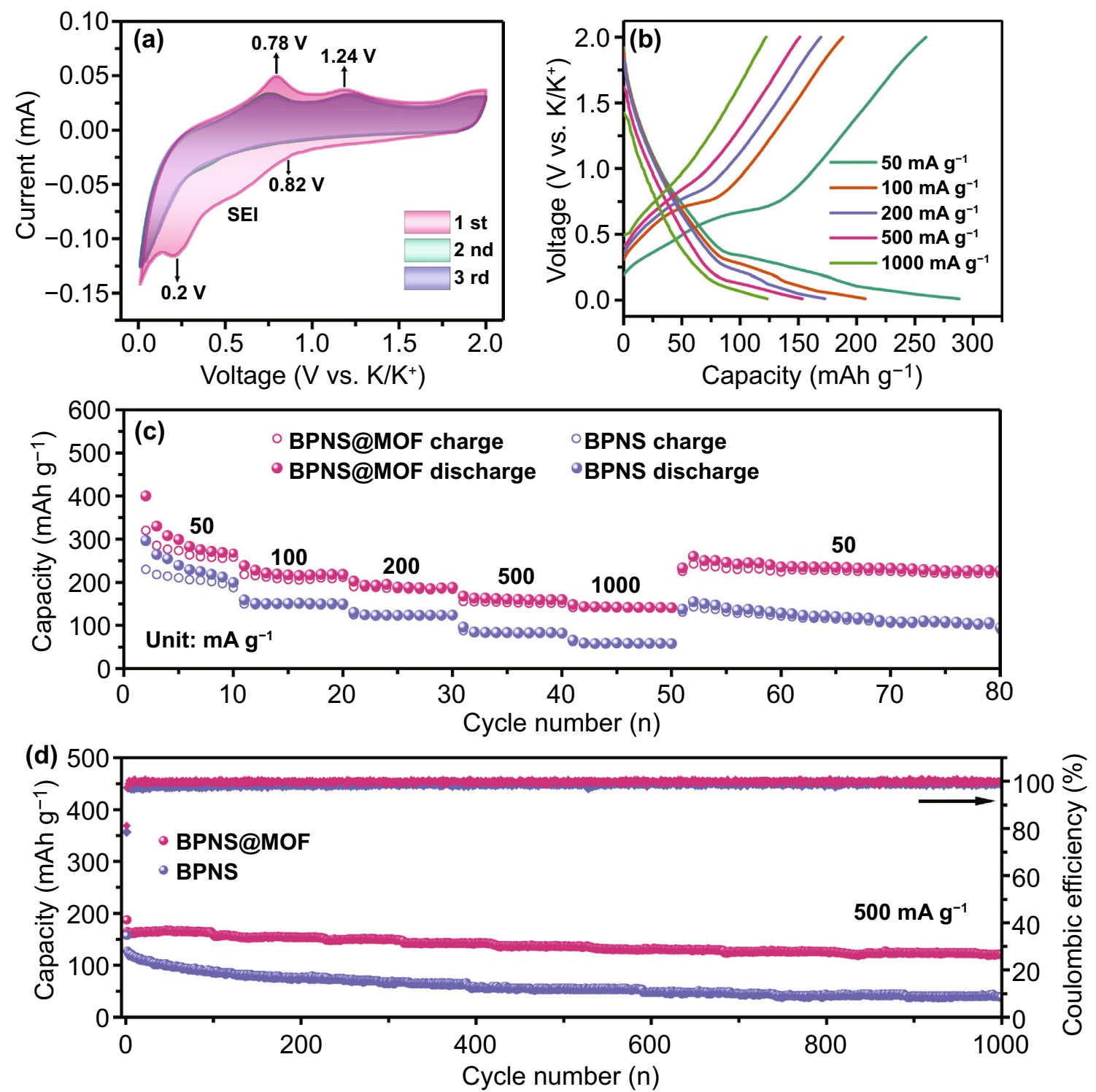

Fig. 4 a CV profiles of the BPNS@MOF electrode at a scan rate of $0.1 \mathrm{mV} \mathrm{s}^{-1}$ for KIB. b Charge-discharge curves of the BPNS@MOF electrode in KIB. $\mathbf{c}$ Rate performance of the BPNS@MOF and BPNS electrodes in KIB at different current densities from 50 to $1000 \mathrm{~mA} \mathrm{~g}^{-1}$. d Cycle performance of the BPNS@MOF and BPNS electrodes in KIB at the current density of $500 \mathrm{~mA} \mathrm{~g}^{-1}$

to the stepwise potassiation process of BP to form the final $\mathrm{K}_{4} \mathrm{P}_{3}$ phase. In the charge process of the BPNS@MOF electrode, an inclined plateau from $0.4-0.75 \mathrm{~V}$ followed by a sloping region up to $2.0 \mathrm{~V}$ corresponds to the stepwise depotassiation reactions of BP. Moreover, the BPNS@MOF electrode manifests better rate capability compared with the BPNS electrode as shown in Fig. 4b, c and Table S2. The capacities achieved for the BPNS@MOF electrode (Fig. 4b) at the same current density are obviously higher than that of the BPNS electrode (Fig. S9). Relatively high average capacities of 301, 222, 192, 162 and $143 \mathrm{mAh} \mathrm{g}^{-1}$ are delivered at respective current densities of 50, 100, 200, 500 and $1000 \mathrm{~mA} \mathrm{~g}^{-1}$ (Fig. 4c, Table S2). Moreover, when the current density finally returns back to $50 \mathrm{~mA} \mathrm{~g}^{-1}$, a capacity of $261 \mathrm{mAh} \mathrm{g}^{-1}$ is still achieved for the BPNS@MOF electrode, with a high capacity retention of $97.2 \%$ (compared with the $10^{\text {th }}$ cycle at $\left.50 \mathrm{~mA} \mathrm{~g}^{-1}\right)$. The values are much higher than that for the BPNS electrode $\left(155 \mathrm{mAh} \mathrm{g}^{-1}\right.$ at $50 \mathrm{~mA} \mathrm{~g}^{-1}$, with a capacity retention of $77.6 \%$ ), manifesting an excellent rate capability of the BPNS@MOF electrode. 
Furthermore, the BPNS@MOF electrode also exhibits excellent cycling stability. The BPNS@MOF electrode can maintain a capacity of $121 \mathrm{mAh} \mathrm{g}^{-1}$ at a higher current density of $500 \mathrm{~mA} \mathrm{~g}^{-1}$ with a high capacity retention of $73 \%$ after 1000 cycles (Fig. 4d). In contrast, the BPNS electrode shows a lower capacity of $43 \mathrm{mAh} \mathrm{g}^{-1}$ as well as a low capacity retention of 34\%. Moreover, the BPNS@MOF electrode still remains electrode integrity after cycles and exhibits a smaller thickness change ratio (33.8\%) than the BPNS electrode (47.1\%) (Fig. S10). The results demonstrate that the MI layer can effectively suppress electrode expansion for stable potassium storage. The performance enhancement is ascribed to the decreased charge transfer resistance at the electrode/electrolyte interface which may facilitate a better charge transfer capability and electrochemical kinetics (Fig. S11, Table S3).

Asymmetric hybrid K-ion capacitors using the BPNS@ MOF or BPNS as the anode and commercial activated carbon (AC, Fig. S12) as the cathode were assembled, as illustrated in Fig. 5a. Based on the voltage range of the CV tests in half-cell configuration with metallic potassium as the counter electrodes (Figs. 4a and S13), the potential window of $0-4.3 \mathrm{~V}$ was selected for the KIC full cell (Fig. 5b). For the KIC assembly, the mass ratio between the AC cathode and BP anode was optimized to be $4: 1$ on a basis of the charge balance theory $\left(\mathrm{Q}^{+}=\mathrm{Q}^{-}\right)$(Fig. S14a). Figure $5 \mathrm{c}$ exhibits the galvanostatic charge-discharge profiles ranging from 50 to $5000 \mathrm{~mA} \mathrm{~g}^{-1}$. The voltage profiles at all current densities are somewhat different from the linear behavior for an ideal supercapacitor, mainly due to the combination of charge storage mechanisms based on faradaic and non-faradaic reactions. The energy/power densities of KICs are shown in a Ragone plot in Fig. 5d. The BPNS@MOF//AC full cell can deliver a high energy density of $93 \mathrm{Wh} \mathrm{kg}^{-1}$ at a power density of $97 \mathrm{~W} \mathrm{~kg}^{-1}$ and a moderate energy density of $48 \mathrm{Wh} \mathrm{kg}^{-1}$ at a high power output of $9380 \mathrm{~W} \mathrm{~kg}^{-1}$. In contrast, BPNS//AC capacitor delivers a maximum energy density of $49 \mathrm{Wh} \mathrm{kg}^{-1}$ at a power density of $102 \mathrm{~W} \mathrm{~kg}^{-1}$ and maintains $22 \mathrm{Wh} \mathrm{kg}^{-1}$ at $92 \mathrm{~W} \mathrm{~kg}^{-1}$, which put up a much lower energy density than BPNS@MOF//AC at a similar power density. The cycle performance of KICs is shown in Fig. 5e, where the BPNS@MOF//AC capacitor can maintain an energy retention of $99.5 \%$ after 6500 cycles at a current density of (a)
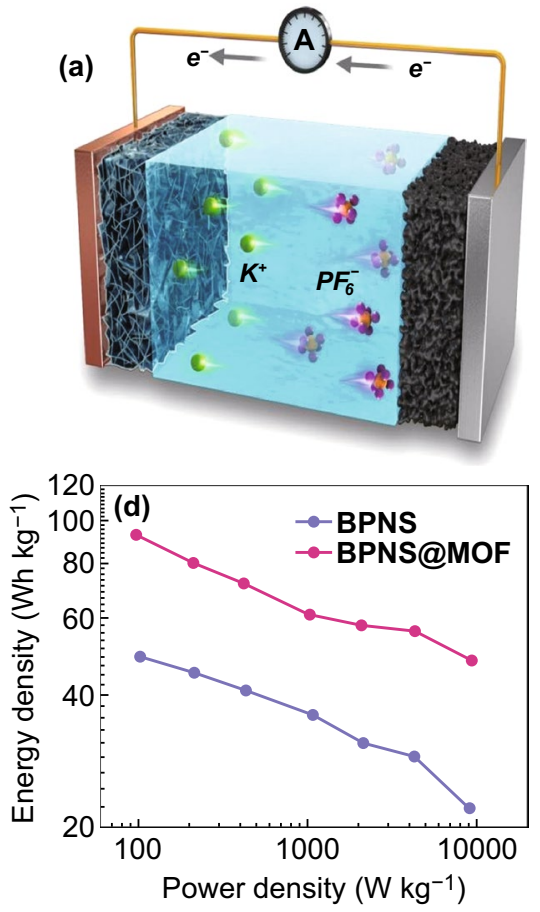
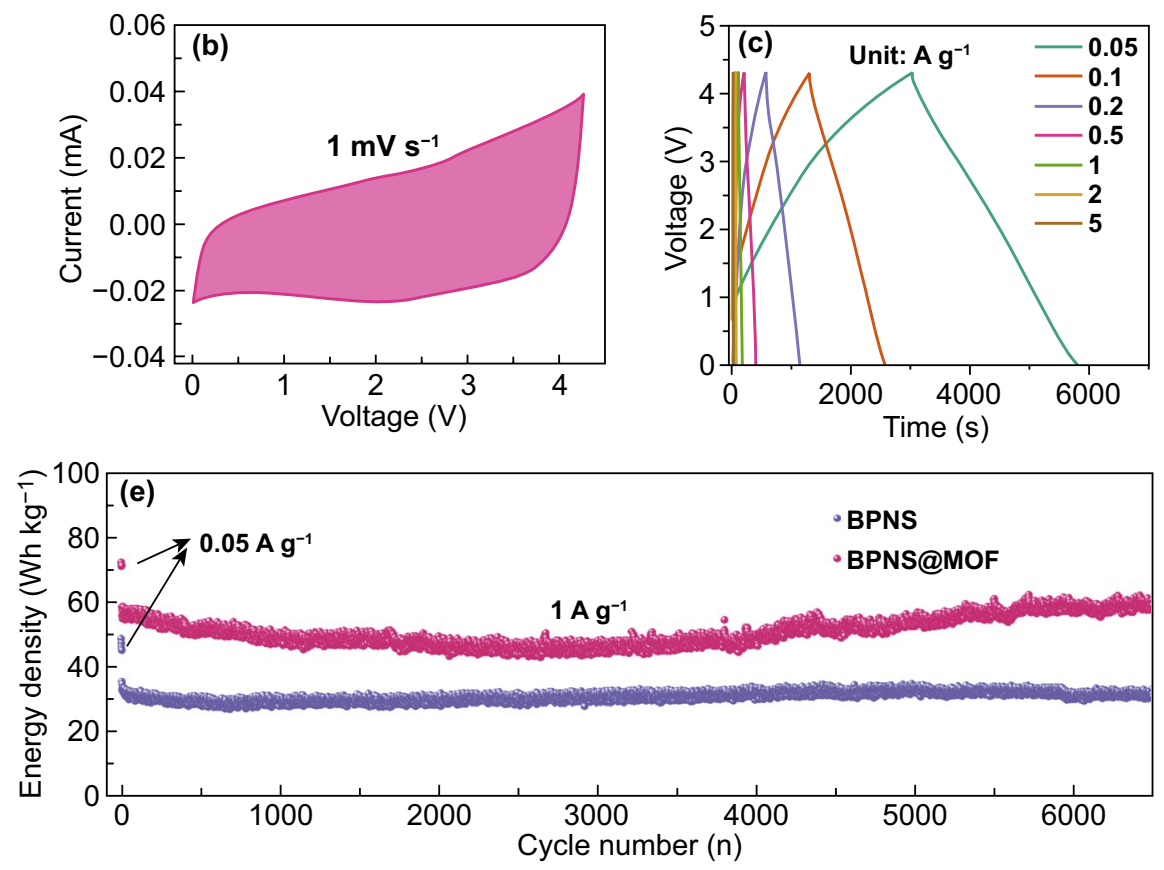

Fig. 5 a Schematic configuration of a KIC device. b CV curve of the BPNS@MOF electrode at scan rate of $1 \mathrm{mV} \mathrm{s}^{-1}$. c Galvanostatic charge/ discharge curves of the BPNS@MOF//AC full cells at different current densities. d Ragone plots of the BPNS//AC and BPNS@MOF//AC electrodes for KIC. e Cyclic performance of the BPNS//AC and BPNS@MOF//AC electrodes for KIC 
$1 \mathrm{~A} \mathrm{~g}^{-1}$, much higher than that of the BPNS//AC capacitor (86\%). Furthermore, BPNS@ MOF samples with different MOF contents were also assembled into KICs and compared their performance (Fig. S14b, c). It is found that their combined energy/power delivery and cycle performance are worse than the typical BPNS@MOF sample. In addition, as shown in Fig. S15, the BPNS@MOF and BPNS@MOF-3 electrodes can remain electrode integrity after cycles due to the relatively thicker MOF interphase layer on BPNSs, while the BPNS@MOF-2 electrode shows obvious cracks on electrode surface after cycles (inset of Fig. S15d1 vs. insets of Fig. S15b1, f1), which may be due to that the thin MOF interphase layer on BPNS cannot effectively suppress electrode expansion. Notably, the BPNS@MOF exhibits a moderate electrode expansion ratio (13.6\%), smaller than that of the BPNS@MOF-2 electrode (18.6\%), yet larger than the BPNS@MOF-2 electrode (10.7\%) (Fig. S15b vs. Fig. S15d, f). The result implies that the typical BPNS@MOF holds an optimal thickness of MOF coating layer to obtain the balance of potassium storage capacity and electrode structural stability. The EIS results for KIC show that the typical BPNS@ MOF sample renders the lowest charge transfer resistance in KIC, which may facilitate a better charge transfer capability and electrochemical kinetics (Fig. S16). Moreover, performance comparison of the BPNS@MOF-based KIC and the state-of-the-art KICs is summarized in Fig. S17. The cycle performance outperforms most of the reported KICs so far (Fig. S17, Table S4). The results suggest that the BPNS@MOF with an ordered porous MI layer stands for an ideal anode material for potassium storage devices.

Moreover, we found that an ultrathin MI layer favors forming a thinner solid electrolyte interphase (SEI) film (3-3.2 nm) on the surface of the BPNS@MOF relative to that of the BPNSs $(6.4-6.8 \mathrm{~nm})$, as shown in Fig. S18. The thinner SEI can facilitate ion insertion/extraction for stable energy storage, as demonstrated in our previous work [45]. Besides, it is analyzed that $\mathrm{K}_{2} \mathrm{SO}_{4}$ is the main component and accounts for the largest percentage among the inorganic components for the BPNS electrode cycled in both KIB and KIC (Figs. S19-S22). The chemical and structural stability of the MI layer in the BPNS@MOF during electrochemical potassium storage was also investigated in depth (Figs. S23 and S24). The result evidences that MI layers can be well preserved upon cycling in KIBs and KICs.

\subsection{Calculation and Analysis of Reaction Kinetics}

To quantitatively analyze the $K$ ion diffusion behavior induced by the MI layer, GITT was employed to determine the reaction kinetics of the BPNS@MOF anodes (Fig. S25). The GITT result demonstrates that the BPNS@MOF electrode can achieve a faster $\mathrm{K}^{+}$migration compared to the BPNS electrode. The increment of the diffusion coefficient may be attributed to the ordered porous structure of the MI layer which provides direct and efficient diffusion pathways of potassium ions during cycles. It is well known that SEI is a passivation layer formed on electrode surfaces from decomposition products of electrolytes [46]. Ideally, the SEI layer should facilitate reversible ions transport and prevent electrode pulverization by suppressing excessive interfacial interactions. However, the SEI layer is normally formed in a dense or nonporous/irregularly porous structure, which is adverse to ion diffusing across to react with electrode materials. For the two systems of the BPNS electrode with the thicker SEI layers and the BPNS@MOF electrode with the thinner SEI and ultrathin MI layers (Fig. S18), the MI layer in the BPNS@MOF in position is analogous to the part of the thicker SEI layer, but may play distinct role in reaction kinetics. To further bring insight into the improved depotassiation/potassiation kinetics by the ordered porous MI in the BPNS@MOF over the corresponding part of SEI on the BPNS electrodes, we carried out calculation of $\mathrm{K}$ diffusion energy barriers for the two systems using density functional theory (DFT) [47]. It is noteworthy that for the BPNS system, $\mathrm{K}_{2} \mathrm{SO}_{4}$ as the main inorganic component of SEI (Figs. S19-S22) was selected to represent the naturally generated SEI to make a comparison with MI layer. The models used for theoretical calculations are illustrated in Fig. S26a, $\mathrm{b}$, where $\mathrm{BP}(020)-\mathrm{MOF}(112)$ interface is employed for the BPNS@MOF, while BP(020)- $\mathrm{K}_{2} \mathrm{SO}_{4}(002)$ is employed for the BPNSs. Calculations of $K$ diffusion energy barriers for the models of both BPNS@MOF and BPNS $/ \mathrm{K}_{2} \mathrm{SO}_{4}$ are divided into two parts, that is, the migration pathway of (i) $K$ ions across $\mathrm{MOF}$ or $\mathrm{K}_{2} \mathrm{SO}_{4}$ layer and (ii) $K$ ions along the surface of BP nanosheet. The optimal migration pathways for the two systems are illustrated in Fig. S26c, d, which are determined by identifying the lowest energy barrier of several possible pathways in MOF layer (Figs. 6a and S27, S28), or the energetically favorable adsorption site in $\mathrm{K}_{2} \mathrm{SO}_{4}$ layer (Figs. $6 \mathrm{~b}$ and S29). It is noted that for the pathway (ii), $K$ along the zigzag direction of BPNSs surface is adopted for the both 
(a)

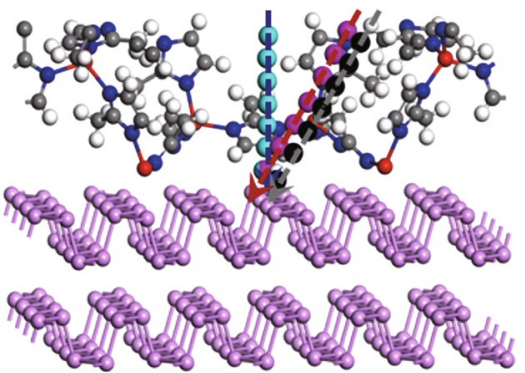

(b)

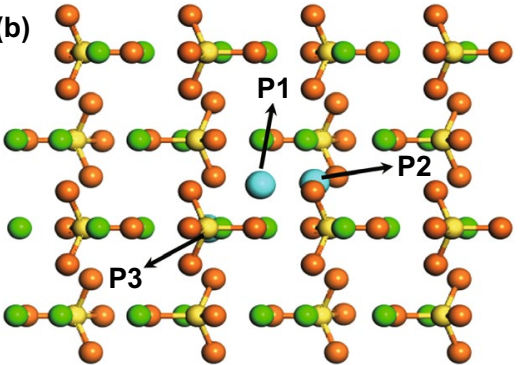

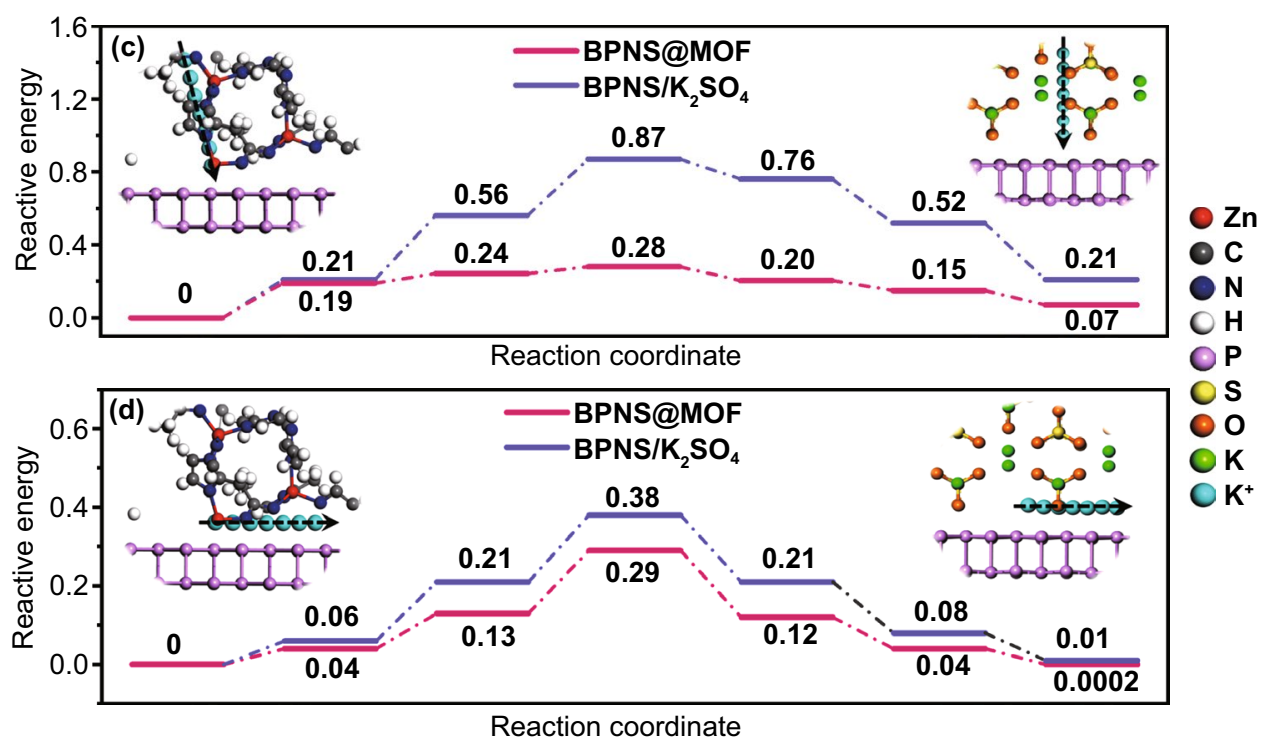

Fig. 6 Theoretical analysis of reaction kinetics. a Different pathways when $\mathrm{K}$ ions pass through MOF in the model of $\mathrm{BP}(020)-\mathrm{MOF}(112)$. b Different sites of $\mathrm{K}$ ions in the model of $\mathrm{BP}(020)-\mathrm{K}_{2} \mathrm{SO}_{4}(002)$. (BPNS is neglected in order to exhibit the site clearly). c Diffusion energy profile for potassium diffusion through the MOF and $\mathrm{K}_{2} \mathrm{SO}_{4}$. Inset of $\mathbf{c}$ is the side views of the diffusion pathways. $\mathbf{d}$ Diffusion energy profile for potassium over the surface of BP. Inset of $\mathbf{d}$ is the side views of the diffusion pathways. Inset of both $\mathbf{c}$ and $\mathbf{d}$ shows $a b$ plane

systems of BPNS@MOF and BPNS/ $\mathrm{K}_{2} \mathrm{SO}_{4}$, which turns out to be the optimum pathway with the lowest diffusion energy barriers [48, 49]. Diffusion energy profiles for $K$ ions diffusion pathway (i) through the MOF or $\mathrm{K}_{2} \mathrm{SO}_{4}$ and (ii) along the surface of BP are shown in Fig. $6 \mathrm{c}$ and $6 \mathrm{~d}$, respectively. The energy barriers of $K$ ion diffusion through BPNS@MOF for pathways (i) and (ii) are both lower than those through BPNS/ $\mathrm{K}_{2} \mathrm{SO}_{4}$. The result indicates that the ordered porous MI layer is more favorable than $\mathrm{K}_{2} \mathrm{SO}_{4}$ for $K$ ions to pass through, thus accounting for a better rate performance.

\section{Conclusions}

In summary, ultrathin MI layers with ordered pores and high stability have been constructed on the surface of the BPNSs, which acting as a protective layer, is proved to play a significant role in improving the electrochemical potassium storage performance. When employed as an anode for KIC, the BPNS@MOF electrode exhibits remarkable performance compared with the pure BPNS electrode. The performance enhancement is ascribed to facilitated $\mathrm{K}$-ion diffusion and reinforced electrode stability enabled by the MI layer with the porous and robust interface. This study provides a new strategy to regulate reaction kinetics and stability of electrode materials through MOF-based interface engineering.

Acknowledgements This work was financially supported by the National Natural Science Foundation (51972235), the Natural Science Foundation of Shanghai (17ZR1447800), Jiangsu Key R\&D Plan (BE2018006-4), the Program for Professor of Special Appointment (Eastern Scholar) at the Shanghai Institutions of Higher Learning and the Fundamental Research Funds for the Central Universities. 
Open Access This article is licensed under a Creative Commons Attribution 4.0 International License, which permits use, sharing, adaptation, distribution and reproduction in any medium or format, as long as you give appropriate credit to the original author(s) and the source, provide a link to the Creative Commons licence, and indicate if changes were made. The images or other third party material in this article are included in the article's Creative Commons licence, unless indicated otherwise in a credit line to the material. If material is not included in the article's Creative Commons licence and your intended use is not permitted by statutory regulation or exceeds the permitted use, you will need to obtain permission directly from the copyright holder. To view a copy of this licence, visit http://creativecommons.org/licenses/by/4.0/.

Electronic supplementary material The online version contains supplementary material available at (https://doi.org/10.1007/ s40820-020-00570-7) contains supplementary material, which is available to authorized users.

\section{References}

1. D. Larcher, J.M. Tarascon, Towards greener and more sustainable batteries for electrical energy storage. Nat. Chem. 7, 19-29 (2015). https://doi.org/10.1038/nchem.2085

2. J.C. Pramudita, D. Sehrawat, D. Goonetilleke, N. Sharma, An initial review of the status of electrode materials for potassium-ion batteries. Adv. Energy Mater. 7, 1602911 (2017). https://doi.org/10.1002/aenm.201602911

3. Z. Jian, W. Luo, X. Ji, Carbon electrodes for K-ion batteries. J. Am. Chem. Soc. 137, 11566-11569 (2015). https://doi. org/10.1021/jacs.5b06809

4. K. Lei, C. Wang, L. Liu, Y. Luo, C. Mu et al., A porous network of bismuth used as the anode material for high-energydensity potassium-ion batteries. Angew. Chem. Int. Ed. 57, 4687-4691 (2018). https://doi.org/10.1002/ange.201801389

5. D. Bin, X. Lin, Y. Sun, Y. Xu, K. Zhang et al., Engineering hollow carbon architecture for high-performance K-ion battery anode. J. Am. Chem. Soc. 140, 7127-7134 (2018). https://doi. org/10.1021/jacs.8b02178

6. L. Fan, K. Lin, J. Wang, R. Ma, B. Lu, A nonaqueous potassium-based battery-supercapacitor hybrid device. Adv. Mater. 30, 1800804 (2018). https://doi.org/10.1002/adma.201800804

7. J. Chen, B. Yang, H. Hou, H. Li, L. Liu et al., Disordered large interlayer spacing and oxygen-rich carbon nanosheets for potassium ion hybrid capacitor. Adv. Energy Mater. 9, 1803894 (2019). https://doi.org/10.1002/aenm201803894

8. M. Shao, C. Li, T. Li, H. Zhao, W. Yu et al., Pushing the energy output and cycling lifespan of potassium-ion capacitor to high level through metal-organic framework derived porous carbon microsheets anode. Adv. Funct. Mater. (2020). https:// doi.org/10.1002/adfm.202006561

9. F. Ming, H. Liang, W. Zhang, J. Ming, Y. Lei et al., Porous MXenes enable high performance potassium ion capacitors. Nano Energy 62, 853-860 (2019). https://doi.org/10.1016/j. nanoen.2019.06.013
10. Z. Zhang, M. Li, Y. Gao, Z. Wei, M. Zhang et al., Fast potassium storage in hierarchical $\mathrm{Ca}_{0.5} \mathrm{Ti}_{2}\left(\mathrm{PO}_{4}\right)_{3} @ \mathrm{C}$ microspheres enabling high-performance potassium-ion capacitors. Adv. Funct. Mater. 28, 1802684 (2018). https://doi.org/10.1002/ adfm.201802684

11. S. Dong, Z. Li, Z. Xing, X. Wu, X. Ji et al., Novel potassiumion hybrid capacitor based on an anode of $\mathrm{K}_{2} \mathrm{Ti}_{6} \mathrm{O}_{13}$ microscaffold. ACS Appl. Mater. Interfaces 10, 15542-15547 (2018). https://doi.org/10.1021/acsami.7b15314

12. Y. Luo, L. Liu, K. Lei, J. Shi, G. Xu et al., A nonaqueous potassium-ion hybrid capacitor enabled by two-dimensional diffusion pathways of dipotassium terephthalate. Chem. Sci. 10, 2048-2052 (2019). https://doi.org/10.1039/C8SC04489A

13. Y. Wang, Z. Zhang, G. Wang, X. Yang, Y. Sui et al., Ultrafine $\mathrm{Co}_{2} \mathrm{P}$ nanorods wrapped by graphene enable a long cycle life performance for a hybrid potassium-ion capacitor. Nanoscale Horiz. 4, 1394-1401 (2019). https://doi.org/10.1039/C9NH0 $0211 \mathrm{~A}$

14. Y. Yi, Z. Sun, C. Li, Z. Tian, C. Lu et al., Designing 3D biomorphic nitrogen-doped $\mathrm{MoSe}_{2}$ /graphene composites toward high-performance potassium-ion capacitors. Adv. Funct. Mater. 30, 1903878 (2020). https://doi.org/10.1002/ adfm.201903878

15. J. Gao, G. Wang, Y. Liu, J. Li, B. Peng et al., Ternary molybdenum sulfoselenide based hybrid nanotubes boost potassiumion diffusion kinetics for high energy/power hybrid capacitors. J. Mater. Chem. A 8, 13946-13954 (2020). https://doi. org/10.1039/d0ta01786h

16. H. Jin, H. Wang, Z. Qi, D.S. Bin, T. Zhang et al., A black phosphorus-graphite composite anode for Li-/Na-/K-ion batteries. Angew. Chem. Int. Ed. 132, 2338-2342 (2020). https ://doi.org/10.1002/ange.201913129

17. J.E.S. Fonsaca, S.H. Domingues, E.S. Orth, A.J.G. Zarbin, A black phosphorus-based cathode for aqueous Na-ion batteries operating under ambient conditions. Chem. Commun. 56, 802 (2020). https://doi.org/10.1039/c9cc09279j

18. Z.L. Xu, S. Lin, N. Onofrio, L. Zhou et al., Exceptional catalytic effects of black phosphorus quantum dots in shuttlingfree lithium sulfur batteries. Nat. Commun. 9, 4164 (2018). https://doi.org/10.1038/s41467-018-06629-9

19. Z. Huang, A. Chen, F. Mo, G. Liang et al., Phosphorene as cathode material for high-voltage, anti-self-discharge zinc ion hybrid capacitors. Adv. Energy Mater. (2020). https://doi. org/10.1002/aenm.202001024

20. L. Li, Y. Yu, J. Ye, Q. Ge, X. Ou et al., Black phosphorus field-effect transistors. Nat. Nanotechnol. 9, 372-377 (2014). https://doi.org/10.1038/NNANO.2014.35

21. J. Cheng, L. Gao, T. Li, S. Mei, C. Wang et al., Two-dimensional black phosphorus nanomaterials: emerging advances in electrochemical energy storage science. Nano-Micro Lett. 12, 179 (2020). https://doi.org/10.1007/s40820-020-00510-5

22. Y. Wu, S. Hu, R. Xu, J. Wang, Z. Peng et al., Boosting potassium-ion battery performance by encapsulating red phosphorus in Free-Standing nitrogen-doped porous hollow carbon nanofibers. Nano Lett. 19, 1351-1358 (2019). https://doi. org/10.1021/acs.nanolett.8b04957 
23. R. Jain, P. Hundekar, T. Deng, X. Fan, Y. Singh et al., Reversible alloying of phosphorene with potassium and its stabilization using reduced graphene oxide buffer layers. ACS Nano 13, 14094-14106 (2019). https://doi.org/10.1021/acsna no. 9 b06680

24. Q. Zhang, J. Mao, W. Pang, T. Zheng, V. Sencadas et al., Boosting the potassium storage performance of alloy-based anode materials via electrolyte salt chemistry. Adv. Energy Mater. 8, 1703288 (2018). https://doi.org/10.1002/aenm.20170 3288

25. Y. An, Y. Tian, L. Ci, S. Xiong, J. Feng et al., Micron-sized nanoporous antimony with tunable porosity for high-performance potassium-ion batteries. ACS Nano 12, 12932-12940 (2018). https://doi.org/10.1021/acsnano.8b08740

26. I. Sultana, M.M. Rahman, Y. Chen, T. Ramireddy, A.M. Glushenkov, Potassium-ion battery anode materials operating through the alloying-dealloying reaction mechanism. Adv. Funct. Mater. 28, 1703857 (2018). https://doi.org/10.1002/ adfm. 201703857

27. J. Huang, S.T. Myung, Y. Sun, Recent progress in rechargeable potassium batteries. Adv. Funct. Mater. 28, 1802938 (2018). https://doi.org/10.1002/adfm.201802938

28. N. Cheng, L. Ren, Y. Xu, Y. Du, S. Dou, Recent development of zeolitic imidazolate frameworks (ZIFs) derived porous carbon based materials as electrocatalysts. Adv. Energy Mater. 8, 1801257 (2018). https://doi.org/10.1002/aenm.201801257

29. J. Liu, D. Zhu, C. Guo, A. Casileff, S. Qiao, Design strategies toward advanced MOF-derived electrocatalysts for energyconversion reactions. Adv. Energy Mater. 7, 1700518 (2017). https://doi.org/10.1002/aenm.201700518

30. L. Fan, Z. Guo, Y. Zhang, X. Wu, C. Zhao et al., Stable artificial solid electrolyte interphase films for lithium metal anode via metal-organic frameworks cemented by polyvinyl alcohol. J. Mater. Chem. A 8, 251-258 (2020). https://doi.org/10.1039/ C9TA10405D

31. B. Li, G. Li, D. Zhang, J. Fan, D. Chen et al., Zeolitic imidazolate framework- 8 modified $\mathrm{LiNi}_{1 / 3} \mathrm{Co}_{1 / 3} \mathrm{Mn}_{1 / 3} \mathrm{O}_{2}$ : A durable cathode showing excellent electrochemical performances in Li-ion batteries. Electrochim. Acta 336, 135724 (2020). https ://doi.org/10.1016/j.electacta.2020.135724

32. X. Liu, F. Yang, W. Xu, Y. Zeng, J. He et al., Zeolitic Imidazolate Frameworks as $\mathrm{Zn}^{2+}$ Modulation Layers to Enable Dendrite-Free Zn Anodes Modulation Layers to Enable DendriteFree Zn Anodes (Sci, Adv, 2020). https://doi.org/10.1002/ advs.202002173

33. M. Zhao, H. Qian, X. Niu, W. Wang, L. Guan et al., Growth mechanism and enhanced yield of black phosphorus microribbons. Cryst. Growth Des. 16, 1096-1103 (2016). https://doi. org/10.1021/acs.cgd.5b01709

34. Z. Huang, H. Hou, Y. Zhang, C. Wang, X. Qiu et al., Layertunable phosphorene modulated by the cation insertion rate as a sodium-storage anode. Adv. Mater. 29, 1702372 (2017). https://doi.org/10.1002/adma.201702372

35. J.P. Perdew, K. Burke, M. Ernzerhof, Generalized gradient approximation made Simple. Phys. Rev. Lett. 77, 3865-3868 (1996). https://doi.org/10.1103/PhysRevLett.77.3865
36. G. Henkelman, H. Jonsson, Improved tangent estimate in the nudged elastic band method for finding minimum energy paths and saddle points. J. Chem. Phys. 113, 9978-9985 (2000). https://doi.org/10.1063/1.1323224

37. B. Xi, Y. Tan, H. Zeng, A general synthetic approach for integrated nanocatalysts of metal-silica@ZIFs. Chem. Mater. 28, 326-336 (2016). https://doi.org/10.1021/acs.chemmater5 b04147

38. R. Meng, J. Huang, Y. Feng, L. Zu, C. Peng et al., Black phosphorus quantum dot/ $/ \mathrm{Ti}_{3} \mathrm{C}_{2}$ MXene nanosheet composites for efficient electrochemical lithium/sodium-ion storage. Adv. Energy Mater. 8, 1801514 (2018). https://doi.org/10.1002/ aenm.201801514

39. S. Gadipelli, W. Travis, W. Zhou, Z. Guo, A thermally derived and optimized structure from $\mathrm{ZIF}-8$ with giant enhancement in $\mathrm{CO}_{2}$ uptake. Energy Environ. Sci. 7, 2232 (2014). https:// doi.org/10.1039/c4ee01009d

40. H. Sobotta, H. Neumann, V. Riede, N.N. Syrbu, Infrared lattice vibration spectra of tetragonal $\mathrm{ZnP}_{2}$. Solid State Commun. 48, 297 (1983). https://doi.org/10.1016/0038-1098(83)90291-0

41. S.R. Venna, J.B. Jasinski, M.A. Carreon, Structural evolution of zeolitic imidazolate framework-8. J. Am. Chem. Soc. 132, 18030-18033 (2010). https://doi.org/10.1021/ja109268m

42. L. Shao, H. Sun, L. Miao, X. Chen, M. Han et al., Facile preparation of $\mathrm{NH}_{2}$-functionalized black phosphorene for the electrocatalytic hydrogen evolution reaction. J. Mater. Chem. A 6, 2494-2499 (2018). https://doi.org/10.1039/C7TA10884B

43. H. Chen, L. Wang, J. Yang, R.T. Yang, Investigation on hydrogenation of metal-organic frameworks HKUST-1, MIL-53 and ZIF-8 by hydrogen spillover. J. Phys. Chem. C 117, 75657576 (2013). https://doi.org/10.1021/jp401367k

44. N. Liedana, A. Galve, C. Rubio, C. Tellez, J. Coronas, CAF@ ZIF-8: One-Step encapsulation of caffeine in MOF. ACS Appl. Mater. Interfaces 4, 5016-5021 (2012). https://doi. org/10.1021/am301365h

45. T. He, J. Feng, J. Ru, Y. Feng, R. Lian et al., Constructing heterointerface of metal atomic layer and amorphous anode material for high-capacity and fast lithium storage. ACS Nano 13, 830-838 (2019). https://doi.org/10.1021/acsnano.8b08344

46. A. Wang, S. Kadam, H. Li, S. Shi, Y. Qi, Review on modeling of the anode solid electrolyte interphase (SEI) for lithiumion batteries. NPJ Comput. Mater. 4, 1-26 (2018). https://doi. org/10.1038/s41524-018-0064-0

47. C. He, J. Zhang, W. Zhang, T. Li, GeSe/BP van der waals heterostructures as promising anode materials for potassiumion batteries. J. Phys. Chem. C 123, 5157-5163 (2019). https ://doi.org/10.1021/acs.jpcc.8b08909

48. Q. Yao, C. Huang, Y. Yuan, Y. Liu, S. Liu et al., Theoretical prediction of phosphorene and nanoribbons as fast-charging Li ion battery anode materials. J. Phys. Chem. C 119, 6923-6928 (2015). https://doi.org/10.1021/acs.jpcc.5b02130

49. V.V. Kulish, O.I. Malyi, C. Persson, P. Wu, C. Persson, Phosphorene as an anode material for Na-ion batteries: a firstprinciples study. Phys. Chem. Chem. Phys. 17, 13921-13928 (2015). https://doi.org/10.1039/C5CP01502B 